\title{
HARMONIC ANALYSIS ON SPHERICAL HOMOGENEOUS SPACES WITH SOLVABLE STABILIZER
}

\author{
ROMAN AVDEEV AND NATALIA GORFINKEL
}

\begin{abstract}
For all spherical homogeneous spaces $G / H$, where $G$ is a simply connected semisimple algebraic group and $H$ a connected solvable subgroup of $G$, we compute the spectra of the representations of $G$ on spaces of regular sections of homogeneous line bundles over $G / H$.
\end{abstract}

\section{INTRODUCTION}

1.1. Let $G$ be a connected semisimple complex algebraic group and let $H$ be a closed subgroup of $G$. One of the problems of harmonic analysis on the homogeneous space $G / H$ is to find the spectrum of the representation of $G$ on the space $\mathbb{C}[G / H]$ of regular functions on $G / H$. An important characteristic of this spectrum is the so-called weight semigroup $\Lambda_{+}(G / H)$. It consists of dominant weights of $G$ such that the space $\mathbb{C}[G / H]$, considered as a $G$-module, contains the irreducible $G$-submodule with highest weight $\lambda$.

The problem described above admits a natural generalization. Namely, one may consider the problem of finding the spectra of the representations of $G$ on spaces of regular sections of homogeneous line bundles over $G / H$. The whole collection of these spectra also determines a semigroup $\widehat{\Lambda}_{+}(G / H)$ called the extended weight semigroup of the homogeneous space $G / H$. The exact definition of this semigroup will be given in $\S 1.2$. The semigroup $\Lambda_{+}(G / H)$ is naturally identified with a subsemigroup of $\widehat{\Lambda}_{+}(G / H)$ (see $\S[1.2)$.

A subgroup $H \subset G$ (resp. a homogeneous space $G / H)$ is said to be spherical if a Borel subgroup $B \subset G$ has an open orbit in $G / H$. In [VK] the following criterion for $H$ to be spherical was proved.

Theorem 1 ([VK, Theorem 1]). (1) A subgroup $H \subset G$ is spherical if and only if for every homogeneous line bundle $L$ over $G / H$ the representation of $G$ on the space of regular sections of $L$ is multiplicity free.

(2) In the case of quasi-affine $G / H$, a subgroup $H \subset G$ is spherical if and only if the representation of $G$ on the space $\mathbb{C}[G / H]$ of regular functions on $G / H$ is multiplicity free.

It follows from this theorem that for a spherical homogeneous space $G / H$ the semigroup $\widehat{\Lambda}_{+}(G / H)$ determines the $G$-module structures on spaces of regular sections of all homogeneous line bundles over $G / H$ and $\Lambda_{+}(G / H)$ determines the $G$-module structure on $\mathbb{C}[G / H]$. In this connection, computation of the semigroups $\Lambda_{+}(G / H)$ and $\widehat{\Lambda}_{+}(G / H)$ for spherical homogeneous spaces $G / H$ is of interest.

2010 Mathematics Subject Classification. 20G05, 22E46, 43A85, 14M27, 14 M17.

Key words and phrases. Algebraic group, homogeneous space, spherical subgroup, representation, semigroup.

Partially supported by Russian Foundation for Basic Research, grant no. 09-01-00648. 
At present, considerable advancements have been achieved in computing weight semigroups for affine spherical homogeneous spaces $G / H$ (that is, with reductive $H$ ). Namely, there is a description of the semigroups $\Lambda_{+}(G / H)$ for all strictly irreducible simply connected affine spherical homogeneous spaces $G / H$ (see their definition, for instance, in Av1, $\S 1.4]$ ), among them are all simply connected affine spherical homogeneous spaces of simple groups. For such spaces $G / H$, in the case of simple $G$ the semigroups $\Lambda_{+}(G / H)$ were computed in $[\mathrm{Kr}]$ and in the case of non-simple $G$ the semigroups $\widehat{\Lambda}_{+}(G / H)$ were computed in Av1. Using these results and simple additional considerations, one can obtain a description of the semigroups $\widehat{\Lambda}_{+}(G / H)$ for all simply connected affine spherical homogeneous spaces $G / H$, however this aspect has not yet been reflected in the literature.

Remark 1. As far as the authors are informed, in the case of non-simple $G$ the semigroups $\widehat{\Lambda}_{+}(G / H)$ for all simply connected strictly irreducible affine spherical homogeneous spaces $G / H$ were computed by Yu. V. Dzyadyk as long ago as in 1985. However these results have not been published.

For non-affine spherical homogeneous spaces $G / H$, the state of the art in the computation of the semigroups $\Lambda_{+}(G / H)$ or $\widehat{\Lambda}_{+}(G / H)$ is much more complicated: the authors are aware of only several particular cases of computing these semigroups. For instance, it is not hard to describe the semigroup $\widehat{\Lambda}_{+}(G / H)$ in the case where $H$ is an intermediate subgroup between some parabolic subgroup of $G$ and its derived subgroup. (Such subgroups $H$ are exactly the horospherical subgroups, that is, subgroups containing some maximal unipotent subgroup of $G$; see $[\mathrm{Kn}, \S 2]$ about that.) Another particular case was examined in Gor, where the semigroups $\widehat{\Lambda}_{+}(G / H)$ were computed in the case where $G$ is simply connected and $H=T U^{\prime}$ ( $U$ is a maximal unipotent subgroup of $G, U^{\prime}$ is its derived subgroup, $T$ is a maximal torus in $G$ normalizing $U$ ).

In the general case, for spherical homogeneous spaces $G / H$ it is more convenient to compute the semigroup $\widehat{\Lambda}_{+}(G / H)$. This is caused by the following reason: if $G$ is simply connected, then $\widehat{\Lambda}_{+}(G / H)$ is free for any spherical subgroup $H \subset G$ (see Theorem 2 in $\S 1.3)$. In this case, to compute the semigroup $\widehat{\Lambda}_{+}(G / H)$ it suffices to find its rank and present the required number of its indecomposable elements. As far as the semigroup $\Lambda_{+}(G / H)$ is concerned, it turns out to be free much more rarely. Namely, for $G$ simply connected and $H$ connected a known sufficient condition for this semigroup to be free is that $H$ has no non-trivial characters (see [Pa1, Proposition 2]). We note that, generally speaking, this condition is quite restrictive.

In the present paper we compute the semigroups $\widehat{\Lambda}_{+}(G / H)$ for all spherical homogeneous spaces $G / H$, where $G$ is a simply connected semisimple group and $H$ is a connected solvable subgroup of $G$. The approach used for this purpose combines ideas contained in Gor and [Av2]. Namely, we generalize the technique applied in Gor for computing the semigroups $\widehat{\Lambda}_{+}(G / H)$ in the case $H=T U^{\prime}$ (see above) to the case of an arbitrary connected solvable spherical subgroup $H \subset G$, making use of a structure theory of connected solvable spherical subgroups in semisimple algebraic groups developed in Av2. The computation results are expressed in terms of combinatorial data considered in [Av2]. The main result of this paper is Theorem 4 (see $\S 2.1$ ).

1.2. Throughout this paper the ground field is the field $\mathbb{C}$ of complex numbers, all topological terms relate to the Zarisky topology, all groups are supposed to be algebraic and 
their subgroups closed. Tangent algebras of groups denoted by capital Latin letters are denoted by the corresponding small German letters. For any group $L$ we denote by $\mathfrak{X}(L)$ the group of its characters in additive notation.

In what follows, $G$ denotes a connected semisimple algebraic group. In $G$ we fix a Borel subgroup $B$ and a maximal torus $T$ contained in $B$. The maximal unipotent subgroup of $G$ contained in $B$ is denoted by $U$. The groups $\mathfrak{X}(B)$ and $\mathfrak{X}(T)$ are identified by restricting characters from $B$ to $T$. The set of dominant weights of $G$ with respect to $B$ is denoted by $\Lambda_{+}(G) ; \Lambda_{+}(G) \subset \mathfrak{X}(B)$. This notation agrees with the notation $\Lambda_{+}(G / H)$ introduced in $\S 1.1$ in the case $H=\{e\}$. For $\lambda \in \Lambda_{+}(G)$ we denote by $V(\lambda)$ the irreducible $G$-module with highest weight $\lambda$ and by $v_{\lambda}$ a fixed highest-weight vector in $V(\lambda)$ (with respect to $B)$. For every $\lambda \in \Lambda_{+}(G)$ the highest weight of the irreducible $G$-module dual to $V(\lambda)$ is denoted by $\lambda^{*}$.

The actions of $G$ on itself by left translation $((g, x) \mapsto g x)$ and right translation $\left((g, x) \mapsto x g^{-1}\right)$ induce representations of $G$ on the space $\mathbb{C}[G]$ of regular functions on $G$ by the formulas $(g f)(x)=f\left(g^{-1} x\right)$ and $(g f)(x)=f(x g)$, respectively $(g, x \in G, f \in \mathbb{C}[G])$. For brevity, we refer to these actions as the action on the left and on the right, respectively (where $g, x \in G$ and $f \in \mathbb{C}[G]$ ). For every subgroup $L \subset G$ we denote by ${ }^{L} \mathbb{C}[G]$ (resp. $\mathbb{C}[G]^{L}$ ) the algebra of functions in $\mathbb{C}[G]$ that are invariant under the action of $L$ on the left (resp. on the right).

Let $H \subset G$ be an arbitrary subgroup. We recall (see [Pop, Theorem 4]) that the homogeneous line bundles over $G / H$ are in one-to-one correspondence with the characters of $H$. Namely, a character $\chi \in \mathfrak{X}(H)$ corresponds to a homogeneous line bundle $L(\chi)=$ $\left(G \times \mathbb{C}_{\chi}\right) / H$ over $G / H$, where $H$ acts on $G$ by right translation and on the space $\mathbb{C}_{\chi} \simeq \mathbb{C}$ by means of the character $\chi$. The fiber of $L(\chi)$ over the point $e H$ is the line $\mathbb{C}_{\chi}$. For every $\chi \in \mathfrak{X}(H)$ there is a natural $G$-equivariant isomorphism between the space $\Gamma(L(-\chi))$ of regular sections of $L(-\chi)$ and the space

$$
V_{\chi}=\{f \in \mathbb{C}[G] \mid f(g h)=\chi(h) f(g) \forall g \in G, \forall h \in H\} \subset \mathbb{C}[G] .
$$

Under this isomorphism, a function $f \in V_{\chi}$ corresponds to the section $\gamma_{f} \in \Gamma(L(-\chi))$ given by the formula $\gamma_{f}(g H)=[g, f(g)]$, where $[g, f(g)]$ is the class in $L(-\chi)$ of the pair $(g, f(g))$. It is easy to see that the space $V_{0} \simeq \Gamma(L(0))$ corresponding to the character $\chi=0$ is nothing else but the space $\mathbb{C}[G]^{H}=\mathbb{C}[G / H]$ of regular functions on $G / H$. We note that $\bigoplus_{\chi \in \mathfrak{X}(H)} V_{\chi}=\mathbb{C}[G]^{H_{0}}$, where the subgroup $H_{0} \subset H$ is the intersection of kernels of all characters of $H$.

Suppose that $\lambda \in \Lambda_{+}(G)$ and $\chi \in \mathfrak{X}(H)$. A function $f \in \mathbb{C}[G]$ is said to be $(B \times H)$ semi-invariant of weight $(\lambda, \chi)$ if $f$ is semi-invariant of weight $\lambda$ under the action of $B$ on the left and semi-invariant of weight $\chi$ under the action of $H$ on the right, that is, $f\left(b^{-1} g h\right)=\lambda(b) \chi(h) f(g)$ for all $b \in B, g \in G, h \in H$. Every non-zero $(B \times H)$ semi-invariant function of weight $(\lambda, \chi)$ is a highest weight vector of some irreducible $G$-submodule in $V_{\chi}$ with highest weight $\lambda$, and vice versa. We denote by $A(\lambda, \chi)$ the subspace in $\mathbb{C}[G]$ consisting of all $(B \times H)$-semi-invariant functions of weight $(\lambda, \chi)$. It is easy to see that the multiplicity with which the irreducible $G$-module with highest weight $\lambda$ occurs in the $G$-module $V_{\chi}$ equals $\operatorname{dim} A(\lambda, \chi)$.

Definition 1. The extended weight semigroup $\widehat{\Lambda}_{+}(G / H)$ of a homogeneous space $G / H$ is the set of pairs $(\lambda, \chi)$ (where $\lambda \in \Lambda_{+}(G)$ and $\chi \in \mathfrak{X}(H)$ ) such that $\operatorname{dim} A(\lambda, \chi) \geqslant 1$. 
The inclusion $A\left(\lambda_{1}, \chi_{1}\right) A\left(\lambda_{2}, \chi_{2}\right) \subset A\left(\lambda_{1}+\lambda_{2}, \chi_{1}+\chi_{2}\right)$ implies that the set $\widehat{\Lambda}_{+}(G / H)$ is indeed a semigroup. We note that this semigroup always contains the element $(0,0)$ since the space $V_{0}=\mathbb{C}[G / H]$ of regular functions on $G / H$ always contains the constants. Thus $\widehat{\Lambda}_{+}(G / H)$ is a monoid.

Evidently, $\Lambda_{+}(G / H) \simeq\left\{(\lambda, \chi) \in \widehat{\Lambda}_{+}(G / H) \mid \chi=0\right\} \subset \widehat{\Lambda}_{+}(G / H)$. In particular, $\Lambda_{+}(G / H) \simeq \widehat{\Lambda}_{+}(G / H)$ whenever $\mathfrak{X}(H)=0$.

We recall that there is the following isomorphism of $(G \times G)$-modules:

$$
\mathbb{C}[G] \simeq \bigoplus_{\lambda \in \Lambda_{+}(G)} V\left(\lambda^{*}\right) \otimes V(\lambda)
$$

on the left-hand side, $G \times G$ acts on the left and on the right, and in each summand on the right-hand side the left (resp. right) factor of $G \times G$ acts on the left (resp. right) tensor factor. We note that for fixed $\lambda \in \Lambda_{+}(G)$ the $(G \times G)$-module $V\left(\lambda^{*}\right) \otimes V(\lambda)$ is embedded in $\mathbb{C}[G]$ as follows: the image of an element $u \otimes v \in V\left(\lambda^{*}\right) \otimes V(\lambda)$ is the function whose value at each point $g \in G$ equals $\langle u, g v\rangle$, where $\langle\cdot, \cdot\rangle$ is the natural pairing between $V\left(\lambda^{*}\right)$ and $V(\lambda)$. Under isomorphism (11) the subspace $A\left(\lambda^{*}, \chi\right) \subset \mathbb{C}[G]$ corresponds to the subspace $v_{\lambda^{*}} \otimes V(\lambda)_{\chi}^{(H)} \subset V\left(\lambda^{*}\right) \otimes V(\lambda)$, where $V(\lambda)_{\chi}^{(H)} \subset V(\lambda)$ is the subspace of $H$-semiinvariant vectors of weight $\chi$. Hence $\operatorname{dim} A\left(\lambda^{*}, \chi\right)=\operatorname{dim} V(\lambda)_{\chi}^{(H)}$ and $\left(\lambda^{*}, \chi\right) \in \widehat{\Lambda}_{+}(G / H)$ if and only if $V(\lambda)_{\chi}^{(H)} \neq 0$.

1.3. We now suppose that $H \subset G$ is a spherical subgroup. In this case, Theorem 1 implies that the condition $\left(\lambda^{*}, \chi\right) \in \widehat{\Lambda}_{+}(G / H)$ is equivalent to either of the two conditions $\operatorname{dim} A\left(\lambda^{*}, \chi\right)=1$ and $\operatorname{dim} V(\lambda)_{\chi}^{(H)}=1$.

An important a priori information about $\widehat{\Lambda}_{+}(G / H)$ is given by the following theorem.

Theorem 2. If $G$ is simply connected, then for every spherical subgroup $H \subset G$ the semigroup $\widehat{\Lambda}_{+}(G / H)$ is free. More precisely, $\widehat{\Lambda}_{+}(G / H)$ is isomorphic to the semigroup $\mathcal{D}(G / H)$ of effective $B$-stable divisors in $G / H$, which is freely generated by the finite set of $B$-stable prime divisors in $G / H$.

In the case of a connected spherical subgroup $H \subset G$ the fact that $\widehat{\Lambda}_{+}(G / H)$ is free can be proved by an argument due to Panyushev, although in his paper [Pa1] he applied it to a more particular situation, namely, to prove that the semigroup $\Lambda_{+}(G / H)$ is free if $\mathfrak{X}(H)=0$ (see [Pa1, Proposition 2]). For an adaptation of this argument to the situation under consideration see [Av1, Theorem 1]. The following proof of Theorem 2 in the general case was communicated to the authors by D. A. Timashev.

Proof of Theorem 2, Regard the map $\rho: \widehat{\Lambda}_{+}(G / H) \rightarrow \mathcal{D}(G / H)$ defined as follows. To each element $(\lambda, \chi) \in \widehat{\Lambda}_{+}(G / H)$ we assign the divisor of zeros of an (arbitrary) non-zero section in the one-dimensional subspace $A(\lambda, \chi) \subset V_{\chi} \simeq \Gamma(L(-\chi))$. It is easy to see that this divisor is $B$-stable and the map $\rho$ is a semigroup homomorphism. Conversely, let $D \in \mathcal{D}(G / H)$ be an arbitrary divisor. Being a Weil divisor on $G / H, D$ is locally principal and hence determines a line bundle $L$ over $G / H$ together with a section $s$ (uniquely determined up to proportionality) in such a way that $D$ is the divisor of $s$. As $D$ is effective, the section $s$ is regular. Further, since $G$ is simply connected, its action on $G / H$ 'lifts' to an action on $L$, that is, the bundle $L$ is homogeneous (see Pop, Proposition 1 
and Theorem 4]). The divisor $D$ is $B$-stable, hence $s$ is $B$-semi-invariant. It follows that $\rho$ is a bijection and thereby an isomorphism.

Remark 2. In the case of simply connected $G$, the algebra $\mathbb{C}[G]$ is factorial (see Pop, Corollary from Proposition 1]); therefore, under the assumptions of Theorem 2, the semigroup $\widehat{\Lambda}_{+}(G / H)$ is also isomorphic to the semigroup of effective $(B \times H)$-stable divisors in $G$. Under this isomorphism an element $(\lambda, \chi) \in \widehat{\Lambda}_{+}(G / H)$ corresponds to the divisor of zeros of some (any) non-zero $(B \times H)$-semi-invariant function in $A(\lambda, \chi)$. In the case of connected $H$ the semigroup of effective $(B \times H)$-stable divisors in $G$ is freely generated by the finite set of prime $(B \times H)$-stable divisors. In this situation, an element $(\lambda, \chi) \in \widehat{\Lambda}_{+}(G / H)$ is indecomposable if and only if the corresponding non-zero $(B \times H)$-semi-invariant function in $A(\lambda, \chi)$ is irreducible in $\mathbb{C}[G]$.

\subsection{Some notation and conventions.}

$e$ is the identity element of any group

$|X|$ is the cardinality of a finite set $X$

$V^{*}$ is the space of linear functions on a vector space $V$

$\operatorname{diag}\left(a_{1}, \ldots, a_{n}\right)$ is the diagonal matrix of order $n$ with elements $a_{1}, \ldots, a_{n}$ on the diagonal

For a group $L$, the notation $L=L_{1} \measuredangle L_{2}$ signifies that $L$ decomposes into a semidirect product of subgroups $L_{1}, L_{2}$, that is, $L=L_{1} L_{2}, L_{1} \cap L_{2}=\{e\}$, and $L_{2}$ is a normal subgroup of $L$.

We identify the lattice $\mathfrak{X}(T)$ with a sublattice in $\mathfrak{t}^{*}$ by associating each character $\mu \in$ $\mathfrak{X}(T)$ with its differential $d \mu \in \mathfrak{t}^{*}$.

In $\mathfrak{X}(T)$ (and thereby in $\mathfrak{t}^{*}$ ), we fix the root system $\Delta$ with respect to $T$ and the set of positive roots $\Delta_{+} \subset \Delta$ with respect to $B$. Let $\Pi=\left\{\alpha_{1}, \ldots, \alpha_{n}\right\} \subset \Delta_{+}$be the set of simple roots and let $\omega_{1}, \ldots, \omega_{n} \in \mathfrak{X}(T) \otimes_{\mathbb{Z}} \mathbb{Q}$ be the fundamental weights corresponding to the simple roots $\alpha_{1}, \ldots, \alpha_{n}$, respectively. In the case of simply connected $G$ one has $\omega_{1}, \ldots, \omega_{n} \in \mathfrak{X}(T)$.

For every root $\alpha \in \sum_{\gamma \in \Pi} k_{\gamma} \gamma \in \Delta_{+}$, we put $\operatorname{Supp} \alpha=\left\{\gamma \mid k_{\gamma}>0\right\}$.

Let $W=N_{G}(T) / T$ be the Weyl group. In the space $\mathfrak{X}(T) \otimes_{\mathbb{Z}} \mathbb{Q}$ we fix an inner product $(\cdot, \cdot)$ invariant under $W$. For $\lambda, \mu \in \mathfrak{X}(T)(\mu \neq 0)$, we put $\langle\lambda \mid \mu\rangle=\frac{2(\lambda, \mu)}{(\mu, \mu)}$.

Let $\Delta^{\vee} \subset \mathfrak{t}$ be the root system dual to $\Delta$. For every $\alpha \in \Delta$ we denote by $h_{\alpha}$ the corresponding element in $\Delta^{\vee}$.

In each root subspace $\mathfrak{g}_{\alpha} \subset \mathfrak{g}$ we choose a basis vector $e_{\alpha}$ in such a way that the condition $\left[e_{\alpha}, e_{-\alpha}\right]=h_{\alpha}$ is fulfilled for every $\alpha \in \Delta$.

Acknowledgements. The authors are deeply grateful to E. B. Vinberg and D. A. Timashev for reading the previous version of this paper and valuable comments.

\section{Formulation of the main Result}

2.1. In order to state the main theorem, we need some facts from the structure theory of connected solvable spherical subgroups in semisimple algebraic groups; see Av2.

Let $H \subset B$ be a connected solvable subgroup and let $N \subset U$ be its unipotent radical. We say that $H$ is standardly embedded in $B$ (with respect to $T$ ) if the subgroup $S=$ 
$H \cap T \subset T$ is a maximal torus in $H$. Obviously, in this situation one has $H=S \wedge N$. Every connected solvable subgroup in $G$ is conjugate by a suitable element of $G$ to a subgroup standardly embedded in $B$.

Suppose that a connected solvable spherical subgroup $H \subset G$ standardly embedded in $B$ is fixed. As above, we put $S=H \cap T$ and $N=H \cap U$ so that $H=S \curlywedge N$. We identify the groups $\mathfrak{X}(H)$ and $\mathfrak{X}(S)$ by restricting characters from $H$ to $S$. We denote by $\tau: \mathfrak{X}(T) \rightarrow \mathfrak{X}(S)$ the character restriction map from $T$ to $S$. Let $\Phi=\tau\left(\Delta_{+}\right) \subset \mathfrak{X}(S)$ be the weight system of the action of $S$ on $\mathfrak{u}$ by means of the adjoint representation of $G$. One has $\mathfrak{u}=\bigoplus_{\varphi \in \Phi} \mathfrak{u}_{\varphi}$, where $\mathfrak{u}_{\varphi} \subset \mathfrak{u}$ is the weight subspace of weight $\varphi$ with respect to $S$. Let $\mathfrak{n}=\bigoplus_{\varphi \in \Phi} \mathfrak{n}_{\varphi}$ be the decomposition of $\mathfrak{n}$ into the direct sum of weight subspaces with respect to $S$. Here for every $\varphi \in \Phi$ one has $\mathfrak{n}_{\varphi} \subset \mathfrak{u}_{\varphi}$. For every $\varphi \in \Phi$ let $c_{\varphi}$ denote the codimension of $\mathfrak{n}_{\varphi}$ in $\mathfrak{u}_{\varphi}$.

In the notation introduced above, there is the following sphericity criterion for a connected solvable subgroup in $G$.

Theorem 3 ([Av2, Theorem 1]). Let $H \subset G$ be a connected solvable subgroup standardly embedded in $B$. The following conditions are equivalent:

(1) $H$ is spherical in $G$;

(2) $c_{\varphi} \leqslant 1$ for every $\varphi \in \Phi$, and all weights with $c_{\varphi}=1$ are linearly independent in $\mathfrak{X}(S)$.

Later on, we assume $H \subset G$ to be a connected solvable spherical subgroup standardly embedded in $B$ and retain the notation introduced above. We put $\Psi=$ $\left\{\alpha \in \Delta_{+} \mid \mathfrak{g}_{\alpha} \not \subset \mathfrak{n}\right\} \subset \Delta_{+}$.

Definition 2. The roots in $\Psi$ are called active.

Let $\varphi_{1}, \ldots, \varphi_{m}$ denote all the weights $\varphi \in \Phi$ with $c_{\varphi}=1$. For $i=1, \ldots, m$ we put $\Psi_{i}=\left\{\alpha \in \Psi \mid \tau(\alpha)=\varphi_{i}\right\}$ and $\mathfrak{u}_{i}=\bigoplus_{\alpha \in \Psi_{i}} \mathfrak{g}_{\alpha}$. It is obvious that $\Psi=\Psi_{1} \cup \Psi_{2} \cup \ldots \cup \Psi_{m}$ and $\mathfrak{u}_{i} \subset \mathfrak{u}_{\varphi_{i}}$.

The set of active roots possesses the following property (see [Av2, Lemma 4]): if $\alpha$ is an active root and $\alpha=\beta+\gamma$ for some $\beta, \gamma \in \Delta_{+}$, then exactly one of the two roots $\beta, \gamma$ is active.

Definition 3. We say that an active root $\beta$ is subordinate to an active root $\alpha$ if there is a root $\gamma \in \Delta_{+}$such that $\alpha=\beta+\gamma$.

Proposition 1 ([Av2, Proposition 3]). Let $\alpha$ be an active root. There is a unique simple root $\pi(\alpha) \in \operatorname{Supp} \alpha$ with the following property: if $\alpha=\alpha_{1}+\alpha_{2}$ for some $\alpha_{1}, \alpha_{2} \in \Delta_{+}$, then $\alpha_{1}\left(\right.$ resp. $\left.\alpha_{2}\right)$ is active if and only if $\pi(\alpha) \notin \operatorname{Supp} \alpha_{1}\left(\right.$ resp. $\left.\pi(\alpha) \notin \operatorname{Supp} \alpha_{2}\right)$.

Thus one has a map $\pi: \Psi \rightarrow \Pi$.

For every $j=1, \ldots, m$, regard the set $\pi\left(\Psi_{j}\right)$. Let $\alpha_{j_{1}}, \alpha_{j_{2}}, \ldots, \alpha_{j_{r}}$, where $r=r(j)=$ $\left|\pi\left(\Psi_{j}\right)\right|$, denote all roots contained in $\pi\left(\Psi_{j}\right)$. We put $\lambda_{j}=\omega_{j_{1}}+\omega_{j_{2}}+\ldots+\omega_{j_{r}}$.

We can now state the main result of the present paper.

Theorem 4. If $G$ is simply connected, then the semigroup $\widehat{\Lambda}_{+}(G / H)$ is freely generated by the elements $\left(\omega_{i}^{*}, \tau\left(\omega_{i}\right)\right), i=1, \ldots, n$, and the elements $\left(\lambda_{j}^{*}, \tau\left(\lambda_{j}\right)-\varphi_{j}\right), j=1, \ldots, m$.

This theorem will be proved in $\S 4$. 
2.2. Let us present two examples of application of Theorem 4. In both cases, the sphericity of $H$ follows from Theorem 3 .

Example 1. Gor Suppose that $G$ is simply connected and $H=T U^{\prime}$. Then $S=T$, $N=U^{\prime}, \mathfrak{n}=\bigoplus_{\alpha \in \Delta_{+} \backslash \Pi} \mathfrak{g}_{\alpha}, \tau=\mathrm{id}, m=n, \Psi=\Pi, \pi=\mathrm{id}$. The semigroup $\widehat{\Lambda}_{+}(G / H)$ is freely generated by the elements $\left(\omega_{i}^{*}, \omega_{i}\right),\left(\omega_{i}^{*}, \omega_{i}-\alpha_{i}\right)$, where $i=1, \ldots, n$.

Example 2. Suppose that $G=\mathrm{SL}_{4}$ and the groups $B, U, T$ consist of all uppertriangular, upper unitriangular, diagonal matrices, respectively, contained in $G$. For $t=\operatorname{diag}\left(t_{1}, t_{2}, t_{3}, t_{4}\right) \in T$ and $k=1,2,3$ we put $\alpha_{k}(t)=t_{k} t_{k+1}^{-1}$. Consider a subgroup $H=S \nless N \subset G$, where $S=\left\{\operatorname{diag}\left(s_{1}, s_{2}, s_{2}^{-1}, s_{1}^{-1}\right) \mid s_{1}, s_{2} \in \mathbb{C}^{\times}\right\}$and $\mathfrak{n} \subset \mathfrak{u}$ is the set of all matrices of the form

$$
\left(\begin{array}{cccc}
0 & a & b & c \\
0 & 0 & d & b \\
0 & 0 & 0 & -a \\
0 & 0 & 0 & 0
\end{array}\right),
$$

where $a, b, c, d \in \mathbb{C}$ are arbitrary numbers. We note that $H$ is a Borel subgroup of the group $\mathrm{Sp}_{4} \subset G$ preserving the skew-symmetric form with matrix

$$
\left(\begin{array}{cccc}
0 & 0 & 0 & 1 \\
0 & 0 & 1 & 0 \\
0 & -1 & 0 & 0 \\
-1 & 0 & 0 & 0
\end{array}\right) .
$$

One has $n=3, m=2, \varphi_{1}=\tau\left(\alpha_{1}\right)=\tau\left(\alpha_{3}\right), \varphi_{2}=\tau\left(\alpha_{1}+\alpha_{2}\right)=\tau\left(\alpha_{2}+\alpha_{3}\right), \Psi_{1}=\left\{\alpha_{1}, \alpha_{3}\right\}$, $\Psi_{2}=\left\{\alpha_{1}+\alpha_{2}, \alpha_{2}+\alpha_{3}\right\}, \pi\left(\alpha_{1}\right)=\alpha_{1}, \pi\left(\alpha_{3}\right)=\alpha_{3}, \pi\left(\alpha_{1}+\alpha_{2}\right)=\pi\left(\alpha_{2}+\alpha_{3}\right)=\alpha_{2}$. For $s=\operatorname{diag}\left(s_{1}, s_{2}, s_{2}^{-1}, s_{1}^{-1}\right) \in S$ we put $\chi_{1}(s)=s_{1}, \chi_{2}(s)=s_{1} s_{2}$. The semigroup $\widehat{\Lambda}_{+}(G / H)$ is freely generated by the five elements $\left(\omega_{3}, \tau\left(\omega_{1}\right)\right)=\left(\omega_{3}, \chi_{1}\right),\left(\omega_{2}, \tau\left(\omega_{2}\right)\right)=\left(\omega_{2}, \chi_{2}\right)$, $\left(\omega_{1}, \tau\left(\omega_{3}\right)\right)=\left(\omega_{1}, \chi_{1}\right),\left(\omega_{1}+\omega_{3}, \tau\left(\omega_{1}+\omega_{3}\right)-\varphi_{1}\right)=\left(\omega_{1}+\omega_{3}, \chi_{2}\right),\left(\omega_{2}, \tau\left(\omega_{2}\right)-\varphi_{2}\right)=\left(\omega_{2}, 0\right)$.

\section{Auxiliary Results}

This section contains all facts needed in the proof of Theorem 4 .

3.1. In this subsection we describe a general approach to computing the rank of the semigroup $\widehat{\Lambda}_{+}(G / H)$ in the case of an arbitrary connected subgroup $H \subset G$. This approach will be applied in $\S 4.1$ to computing the rank of $\widehat{\Lambda}_{+}(G / H)$ under the assumptions of Theorem 4 .

We first recall the following notion.

Let $L$ be a reductive group and let $B_{L}$ be a Borel subgroup of $L$. The rank of the action $L: X$ of $L$ on an irreducible variety $X$ is the rank of the lattice $\Lambda(X) \subset \mathfrak{X}\left(B_{L}\right)$, where $\Lambda(X)$ consists of weights $\mu \in \mathfrak{X}\left(B_{L}\right)$ such that the field $\mathbb{C}(X)$ of rational functions on $X$ contains a non-zero $B_{L}$-semi-invariant function of weight $\mu$. We denote by $r_{L}(X)$ the rank of the action $L: X$. By the rank of a homogeneous space $L / K$ we shall mean the rank of the natural action $L: L / K$ by left translation.

Let $H$ be an arbitrary connected subgroup of $G$. Regard the homogeneous space $G / H_{0}$ (see the definition of the subgroup $H_{0}$ in $\left.\S 1.2\right)$. It is quasi-affine since $H_{0}$ has no non-trivial characters. There is a transitive action of the group $\widehat{G}=G \times H / H_{0}$ on $G / H_{0}$, where 
$G$ acts on the left and $H / H_{0}$ acts on the right. Under this action, the stabilizer of the point $e H_{0}$ is the subgroup $\widehat{H}=\left\{\left(h, h H_{0}\right) \mid h \in H\right\} \subset \widehat{G}$, which is isomorphic to $H$. Thus there is an isomorphism of varieties $G / H_{0} \simeq \widehat{G} / \widehat{H}$. The algebras $\mathbb{C}\left[G / H_{0}\right]$ and $\mathbb{C}[\widehat{G} / \widehat{H}]$ are isomorphic as $\widehat{G}$-modules, hence there is a semigroup isomorphism $\widehat{\Lambda}_{+}(G / H) \simeq \Lambda_{+}(\widehat{G} / \widehat{H})$. Since $\widehat{G} / \widehat{H}$ is quasi-affine, the lattice $\Lambda(\widehat{G} / \widehat{H})$ is generated by the semigroup $\Lambda_{+}(\widehat{G} / \widehat{H})$ (see, for instance, Tim, Proposition 5.14]), therefore $\operatorname{rk} \Lambda_{+}(\widehat{G} / \widehat{H})=\operatorname{rk} \Lambda(\widehat{G} / \widehat{H})$. Hence $\operatorname{rk} \widehat{\Lambda}_{+}(G / H)=r_{\widehat{G}}(\widehat{G} / \widehat{H})$. The rank of the homogeneous space $\widehat{G} / \widehat{H}$ can be computed by using the following general result of Panyushev.

Proposition 2 ([Pa2, Theorem 1.2(ii)]). Let $L$ be a connected reductive group and let $P \subset L$ be a parabolic subgroup with Levi decomposition $P=P_{r} \wedge P_{u}$, where $P_{r}$ and $P_{u}$ are a maximal reductive subgroup and the unipotent radical of $P$, respectively. Let $K \subset L$ be a connected subgroup with Levi decomposition $K=K_{r} \wedge K_{u}$, where $K_{r}$ and $K_{u}$ are a maximal reductive subgroup and the unipotent radical of $K$, respectively, with $K_{r} \subset P_{r}$ and $K_{u} \subset P_{u}$. Finally, let $F \subset K_{r}$ be a generic stabilizer for the action $K_{r}: \mathfrak{p}_{r} / \mathfrak{k}_{r}$ (the subgroup $F$ is reductive $)$. Then $r_{L}(L / K)=r_{P_{r}}\left(P_{r} / K_{r}\right)+r_{F}\left(P_{u} / K_{u}\right)$.

3.2. In this subsection we collect all necessary results of active root theory.

Proposition 3. Suppose that $1 \leqslant i, j \leqslant m$ and different roots $\alpha \in \Psi_{i}, \beta \in \Psi_{j}$ are such that $\gamma=\beta-\alpha \in \Delta_{+}$. Then

(a) [Av2, Proposition 1] $\Psi_{i}+\gamma \subset \Psi_{j}$;

(b) [Av2, Proposition 10(c)] if $\left|\Psi_{i}\right| \geqslant 2$, then $\gamma$ is a unique positive root with the property $\Psi_{i}+\gamma \subset \Psi_{j}$.

Corollary 1. Suppose that $1 \leqslant i \leqslant m$ and roots $\alpha, \beta \in \Psi_{i}$ are different. Then $\alpha-\beta \notin \Delta$.

Proof. Assume that $\gamma=\alpha-\beta \in \Delta$. We may also assume that $\gamma \in \Delta_{+}$. Then in view of Proposition 3(a) one has $\Psi_{i}+\gamma \subset \Psi_{i}$, which is impossible.

For every $i=1, \ldots, m$ the subspace $\mathfrak{n} \cap \mathfrak{u}_{i}$ is determined inside $\mathfrak{u}_{i}$ by the vanishing of a linear function (uniquely determined up to proportionality), which will be denoted by $\xi_{i}$. If $\alpha \in \Psi_{i}$ for some $i \in\{1, \ldots, m\}$, then the restriction of $\xi_{i}$ to $\mathfrak{g}_{\alpha}$ is not zero.

Proposition 4 ([Av2, Proposition 2]). If $\Psi_{i}+\gamma \subset \Psi_{j}$ for some $i, j \in\{1, \ldots, m\}$ and $\gamma \in \Delta_{+}$, then there exists a number $c \neq 0$ such that $\xi_{i}(x)=c \xi_{j}\left(\left[x, e_{\gamma}\right]\right)$ for all $x \in \mathfrak{u}_{i}$.

Proposition 5 (Av2, Corollary 11]). Let active roots $\alpha, \beta$ be such that $\tau(\alpha)=\tau(\beta)$. Then either $\pi(\alpha)=\pi(\beta)$ or none of the roots $\pi(\alpha), \pi(\beta)$ is contained in $\operatorname{Supp} \alpha \cap \operatorname{Supp} \beta$.

\section{Proof of the main theorem}

In view of Theorem 2 the proof of Theorem 4 involves three stages. First, in $\$ 4.1$ we compute the rank of the semigroup $\widehat{\Lambda}_{+}(G / H)$. Then in $\S 4.2$ we show that all the elements mentioned in the statement of Theorem 4 are indeed contained in this semigroup. At last, in $\S 4.3$ we prove that these elements are indecomposable in $\widehat{\Lambda}_{+}(G / H)$.

In this section we preserve the notation introduced in $\S 2.1$. 
4.1. In this subsection we prove the following proposition.

Proposition 6. Under the assumptions of Theorem 4 , the rank of $\widehat{\Lambda}_{+}(G / H)$ equals $n+m$.

Below we present two different proofs of this proposition. The first one uses the general approach for computing the rank of extended weight semigroups, which was described in $\S 3.1$. The second proof is direct and uses a geometric argument.

Proof 1. The natural epimorphism $H \rightarrow H / H_{0}$ maps the subgroup $S \subset H$ isomorphically onto $H / H_{0}$. For this reason, further we identify $S$ and $H / H_{0}$. It was shown in $\S 3.1$ that $\operatorname{rk} \widehat{\Lambda}_{+}(G / H)=r_{\widehat{G}}(\widehat{G} / \widehat{H})$. To compute $r_{\widehat{G}}(\widehat{G} / \widehat{H})$ we apply Proposition 2 with $L=\widehat{G}=$ $G \times S$ and $K=\widehat{H}$. Let $P=B \times S$. Then $P_{r}=T \times S, P_{u}=U ; K_{r}=\widehat{S}, K_{u}=N$, where the subgroup $\widehat{S} \subset \widehat{H}$ is isomorphic to $S$ and embedded diagonally in the subgroup $S \times S \subset H \times S$. Since $\widehat{S}$ acts trivially on $\mathfrak{p}_{r} / \mathfrak{k}_{r} \simeq(\mathfrak{t} \oplus \mathfrak{s}) / \widehat{\mathfrak{s}}$, one has $F=\widehat{S}$. Therefore $r_{\widehat{G}}(\widehat{G} / \widehat{H})=r_{T \times S}((T \times S) / \widehat{S})+r_{\widehat{S}}(U / N)$. It is easy to see that the first summand in the last sum is equal to $\operatorname{rk} T=n$. Let us find the value of the second summand. Clearly, this value coincides with $r_{S}(U / N)$. In view of [Mon, Lemma 1.4] there is an $S$-equivariant isomorphism $U / N \simeq \mathfrak{u} / \mathfrak{n}$. By Theorem 3, $\mathfrak{u} / \mathfrak{n}$ is isomorphic as an $S$-module to the direct sum $\mathbb{C}_{\varphi_{1}} \oplus \ldots \oplus \mathbb{C}_{\varphi_{m}}$, where $\mathbb{C}_{\varphi_{i}} \simeq \mathbb{C}$ is the subspace of weight $\varphi_{i}$ with respect to $S$ and the weights $\varphi_{1}, \ldots, \varphi_{m}$ are linearly independent. This implies that $r_{S}(U / N)=m$.

Proof 2. Since $H$ is connected, in view of Remark 2 the rank of $\widehat{\Lambda}_{+}(G / H)$ equals the number of $(B \times H)$-stable prime divisors in $G$. Regard the Bruhat decomposition of $G$ :

$$
G=\bigsqcup_{\sigma \in W} B \sigma B
$$

where the union is disjoint. Among the subsets $B \sigma B \subset G$, each of them being $(B \times B)$ stable, there is the open subset $G_{0}=B \sigma_{0} B \subset G$ (referred to as the open cell), where $\sigma_{0}$ is the longest element in $W$. It is well known that the complement of $G_{0}$ in $G$ is the union of exactly $n$ prime divisors, which are $(B \times B)$-stable (and so $(B \times H)$-stable). Hence to complete the proof it remains to show that the number of $(B \times H)$-stable prime divisors in $G_{0}$ equals $m$. In view of the isomorphism $G_{0} \simeq B \sigma_{0} \times U$ we obtain that the $(B \times H)$-stable divisors in $G_{0}$ are in one-to-one correspondence with the $H$-stable divisors in $U$, where the action of $H$ on $U$ is given by the formula $(s v, u) \mapsto s u v^{-1} s^{-1}(s \in S$, $v \in N, u \in U)$. In turn, the $H$-stable divisors in $U$ are in one-to-one correspondence with the $S$-stable divisors in $U / N$ or, in view of an $S$-equivariant isomorphism $U / N \simeq \mathfrak{u} / \mathfrak{n}$ (see [Mon, Lemma 1.4]), with the $S$-stable divisors in $\mathfrak{u} / \mathfrak{n}$. Taking into account an $S$ module isomorphism $\mathfrak{u} / \mathfrak{n} \simeq \mathbb{C}_{\varphi_{1}} \oplus \ldots \oplus \mathbb{C}_{\varphi_{m}}$ (see Theorem $[3$ ), we see that the $S$-stable prime divisors in $\mathfrak{u} / \mathfrak{n}$ are exactly the divisors of zeros of $S$-semi-invariant linear functions on $\mathfrak{u} / \mathfrak{n}$. Evidently, up to proportionality there are exactly $m$ such linear functions.

4.2. In this subsection we show that the elements listed in the statement of Theorem 4 are contained in the semigroup $\widehat{\Lambda}_{+}(G / H)$.

As was mentioned earlier ( see $\S 1.2)$, the condition $\left(\lambda^{*}, \chi\right) \in \widehat{\Lambda}_{+}(G / H)$ is equivalent to the condition $V(\lambda)_{\chi}^{(H)} \neq 0$. For the elements $\left(\omega_{i}^{*}, \tau\left(\omega_{i}\right)\right), i=1, \ldots, n$, it is quite easy to point out a non-zero vector in $V\left(\omega_{i}\right)_{\tau\left(\omega_{i}\right)}^{(H)}$ : this is the highest weight vector $v_{\omega_{i}} \in V\left(\omega_{i}\right)$. Now it remains to prove that $\left(\lambda_{j}^{*}, \tau\left(\lambda_{j}\right)-\varphi_{j}\right) \in \widehat{\Lambda}_{+}(G / H)$ or, equivalently, $V\left(\lambda_{j}\right)_{\tau\left(\lambda_{j}\right)-\varphi_{j}}^{(H)} \neq$ 0 for all $j=1, \ldots, m$. This is the objective of the rest of the subsection. 
Below we shall need the following simple lemma.

Lemma 1. Suppose that $\mu \in \Lambda_{+}(G)$ and $\alpha, \beta \in \Delta_{+}$. Then:

(a) $e_{\alpha}\left(e_{-\beta} v_{\mu}\right)=\left[e_{\alpha}, e_{-\beta}\right] v_{\mu}$;

(b) if $\left[e_{\alpha}, e_{-\beta}\right] v_{\mu} \neq 0$, then $\beta-\alpha \in \Delta_{+}$.

We fix $j \in\{1, \ldots, m\}$ and denote by $\beta_{1}, \ldots, \beta_{p}$ all roots in $\Psi_{j}$. The linear function $\xi_{j} \in \mathfrak{u}_{j}^{*}$ (see $\left.\S 3.2\right)$ is determined by the set of non-zero numbers $a_{1}, \ldots, a_{p}$ as follows: an element $x_{1} e_{\beta_{1}}+\ldots+x_{p} e_{\beta_{p}} \in \mathfrak{u}_{j}$ lies in $\mathfrak{n}$ if and only if $a_{1} x_{1}+\ldots+a_{p} x_{p}=0$.

Suppose that $\pi\left(\Psi_{j}\right)=\left\{\alpha_{j_{1}}, \ldots, \alpha_{j_{r}}\right\}$ and $\lambda_{j}=\omega_{j_{1}}+\ldots+\omega_{j_{r}} \in \Lambda_{+}(G)$ (see $\S 2.1$ ). We note that for every $k=1, \ldots, p$ one has $\lambda_{j}\left(h_{\beta_{k}}\right)=\left\langle\lambda_{j} \mid \beta_{k}\right\rangle>0$ in view of the condition $\pi\left(\beta_{k}\right) \in \operatorname{Supp} \beta_{k}$, whence $e_{-\beta_{k}} v_{\lambda_{j}} \neq 0$. We set

$$
f_{j}=\frac{a_{1}}{\lambda_{j}\left(h_{\beta_{1}}\right)} e_{-\beta_{1}}+\ldots+\frac{a_{p}}{\lambda_{j}\left(h_{\beta_{p}}\right)} e_{-\beta_{p}} \in \mathfrak{g}
$$

and regard the (non-zero) vector $w_{j}=f_{j} v_{\lambda_{j}} \in V\left(\lambda_{j}\right)$.

Our goal is to show that $w_{j} \in V\left(\lambda_{j}\right)_{\tau\left(\lambda_{j}\right)-\varphi_{j}}^{(H)}$. It is easy to see that the vector $w_{j}$ is $S$-semi-invariant of weight $\tau\left(\lambda_{j}\right)-\varphi_{j}$. Let us prove that $w_{j}$ is $H$-semi-invariant. For this purpose, it suffices to prove the following proposition.

Proposition 7. The vector $w_{j}$ is annihilated by the algebra $\mathfrak{n}$.

Proof consists of three steps.

Step 1. Let us prove that $w_{j}$ is annihilated by $\mathfrak{n} \cap \mathfrak{u}_{j}$. For this purpose, we take an arbitrary element $x=x_{1} e_{\beta_{1}}+\ldots+x_{p} e_{\beta_{p}} \in \mathfrak{n} \cap \mathfrak{u}_{j}$ and show that $x w_{j}=0$. In view of Lemma 1(a) one has

$$
x w_{j}=\left(\sum_{k=1}^{p} x_{k} e_{\beta_{k}}\right)\left(\sum_{l=1}^{p} \frac{a_{l}}{\lambda_{j}\left(h_{\beta_{l}}\right)} e_{-\beta_{l}}\right) v_{\lambda_{j}}=\sum_{k=1}^{p} \sum_{l=1}^{p}\left(\frac{a_{l} x_{k}}{\lambda_{j}\left(h_{\beta_{l}}\right)}\left[e_{\beta_{k}}, e_{-\beta_{l}}\right] v_{\lambda_{j}}\right) .
$$

By Corollary 1, for all $k \neq l$ one has $\beta_{k}-\beta_{l} \notin \Delta$, whence $\left[e_{\beta_{k}}, e_{-\beta_{l}}\right]=0$. Therefore

$$
x w_{j}=\sum_{k=1}^{p}\left(\frac{a_{k} x_{k}}{\lambda_{j}\left(h_{\beta_{k}}\right)}\left[e_{\beta_{k}}, e_{-\beta_{k}}\right] v_{\lambda_{j}}\right)=\sum_{k=1}^{p}\left(\frac{a_{k} x_{k}}{\lambda_{j}\left(h_{\beta_{k}}\right)} h_{\beta_{k}} v_{\lambda_{j}}\right)=\left(\sum_{k=1}^{p} a_{k} x_{k}\right) v_{\lambda_{j}}=0,
$$

where the relations $h_{\beta_{k}} v_{\lambda_{j}}=\lambda_{j}\left(h_{\beta_{k}}\right) v_{\lambda_{j}}$ and $a_{1} x_{1}+\ldots+a_{p} x_{p}=0$ are taken into account.

Step 2. Let us prove that $w_{j}$ is annihilated by $\mathfrak{n} \cap \mathfrak{u}_{i}$ for $i \neq j$. Suppose that $\Psi_{i}=$ $\left\{\gamma_{1}, \ldots, \gamma_{q}\right\}$. If $\left|\Psi_{i}\right|=1$, then $\mathfrak{n} \cap \mathfrak{u}_{i}=\{0\}$ and there is nothing to prove. So further we assume $\left|\Psi_{i}\right| \geqslant 2$. The linear function $\xi_{i} \in \mathfrak{u}_{i}^{*}$ is determined by a set of non-zero numbers $b_{1}, \ldots, b_{q}$ so that an element $y_{1} e_{\gamma_{1}}+\ldots+y_{q} e_{\gamma_{q}}$ lies in $\mathfrak{n}$ if and only if $b_{1} y_{1}+\ldots+b_{q} y_{q}=0$. Suppose $y=y_{1} e_{\gamma_{1}}+\ldots+y_{q} e_{\gamma_{q}} \in \mathfrak{n} \cap \mathfrak{u}_{i}$. Let us show that $y w_{j}=0$. In view of Lemma 1(a) we have

$$
y w_{j}=\left(\sum_{k=1}^{q} y_{k} e_{\gamma_{k}}\right)\left(\sum_{l=1}^{p} \frac{a_{l}}{\lambda_{j}\left(h_{\beta_{l}}\right)} e_{-\beta_{l}}\right) v_{\lambda_{j}}=\sum_{k=1}^{q} \sum_{l=1}^{p}\left(\frac{a_{l} y_{k}}{\lambda_{j}\left(h_{\beta_{l}}\right)}\left[e_{\gamma_{k}}, e_{-\beta_{l}}\right] v_{\lambda_{j}}\right) .
$$

Assume that for some $k \in\{1, \ldots, q\}$ and $l \in\{1, \ldots, p\}$ the element $\left[e_{\gamma_{k}}, e_{-\beta_{l}}\right]$ acts non-trivially on $v_{\lambda_{j}}$. Then by lemma 1(b) we get $\gamma_{k}-\beta_{l}=-\delta$ for some $\delta \in \Delta_{+}$. In view of Proposition 3(a) one has $\Psi_{i}+\delta \subset \Psi_{j}$. Since $\left|\Psi_{i}\right| \geqslant 2$, by Proposition 3 (b) the root $\delta$ is 
the only positive root with property $\Psi_{i}+\delta \subset \Psi_{j}$. Renumbering the roots in $\Psi_{j}$, without loss of generality we may assume that $\gamma_{1}+\delta=\beta_{1}, \ldots, \gamma_{q}+\delta=\beta_{q}$. Then

$$
y w_{j}=\sum_{k=1}^{q}\left(\frac{a_{k} y_{k}}{\lambda_{j}\left(h_{\beta_{k}}\right)}\left[e_{\gamma_{k}}, e_{-\beta_{k}}\right] v_{\lambda_{j}}\right)=\left(\sum_{k=1}^{q} \frac{a_{k} d_{k} y_{k}}{\lambda_{j}\left(h_{\beta_{k}}\right)}\right) e_{-\delta} v_{\lambda_{j}},
$$

where for $k=1, \ldots, q$ the numbers $d_{k}$ are such that $\left[e_{\gamma_{k}}, e_{-\beta_{k}}\right]=d_{k} e_{-\delta}$. Further, for $k=1, \ldots, q$ one has $\left[e_{\gamma_{k}}, e_{\delta}\right]=c_{k} e_{\beta_{k}}$, where $c_{k} \neq 0$. In view of Proposition 4 we may assume that $b_{k}=a_{k} c_{k}$ for all $k=1, \ldots, q$.

To complete the proof, it suffices to show that

$$
\frac{a_{1} d_{1} y_{1}}{\lambda_{j}\left(h_{\beta_{1}}\right)}+\ldots+\frac{a_{q} d_{q} y_{q}}{\lambda_{j}\left(h_{\beta_{q}}\right)}=0 .
$$

Substituting $a_{k}=\frac{b_{k}}{c_{k}}$ into this formula for $k=1, \ldots, q$ and taking into account the condition $b_{1} y_{1}+\ldots+b_{q} y_{q}=0$, we find that now it suffices to prove the relation

$$
\frac{d_{1}}{c_{1} \lambda_{j}\left(h_{\beta_{1}}\right)}=\ldots=\frac{d_{q}}{c_{q} \lambda_{j}\left(h_{\beta_{q}}\right)} \text {. }
$$

To this end, for fixed $k \in\{1, \ldots, q\}$ we regard the three elements $e_{\gamma_{k}}, e_{\delta}, e_{-\beta_{k}}$ and write down the Jacobi identity for them:

$$
\left[\left[e_{\gamma_{k}}, e_{\delta}\right], e_{-\beta_{k}}\right]+\left[\left[e_{-\beta_{k}}, e_{\gamma_{k}}\right], e_{\delta}\right]+\left[\left[e_{\delta}, e_{-\beta_{k}}\right], e_{\gamma_{k}}\right]=0,
$$

or

$$
c_{k} h_{\beta_{k}}+d_{k} h_{\delta}+A_{k} h_{\gamma_{k}}=0,
$$

where $A_{k}$ is some number. Acting on $v_{\lambda_{j}}$ by both sides of this equality, we obtain

$$
c_{k} \lambda_{j}\left(h_{\beta_{m}}\right)+d_{k} \lambda_{j}\left(h_{\delta}\right)+A_{k} \lambda_{j}\left(h_{\gamma_{k}}\right)=0 .
$$

Since $\gamma_{k}$ is an active root subordinate to the active root $\beta_{k}$, it follows that the simple root $\pi\left(\beta_{k}\right)=\alpha_{j_{l}}$ is not contained in the set $\operatorname{Supp} \gamma_{k}$, whence $\omega_{j_{l}}\left(h_{\gamma_{k}}\right)=0$. Further, in view of condition Supp $\gamma_{m} \subset \operatorname{Supp} \beta_{m}$ and Proposition 5 we obtain $\pi\left(\Psi_{j}\right) \cap \operatorname{Supp} \gamma_{k}=\varnothing$. Hence $\omega_{j_{1}}\left(h_{\gamma_{k}}\right)=\ldots=\omega_{j_{r}}\left(h_{\gamma_{k}}\right)=0$ and $\lambda_{j}\left(h_{\gamma_{k}}\right)=0$. Thus, $c_{k} \lambda_{j}\left(h_{\beta_{k}}\right)+d_{k} \lambda_{j}\left(h_{\delta}\right)=0$. We note that $\lambda_{j}\left(h_{\delta}\right)=\left\langle\lambda_{j} \mid \delta\right\rangle=\left\langle\omega_{j_{l}} \mid \delta\right\rangle>0$ because $\alpha_{j_{l}} \in \operatorname{Supp} \delta$. Hence the quantity

$$
\frac{d_{k}}{c_{k} \lambda_{j}\left(h_{\beta_{k}}\right)}=-\frac{1}{\lambda_{j}\left(h_{\delta}\right)}
$$

is independent of $k$, as required.

Step 3. Let us prove that $w_{j}$ is annihilated by $\mathfrak{g}_{\gamma}$ for every $\gamma \in \Delta_{+} \backslash \Psi$. In view of Lemma 1(a) we have

$$
e_{\gamma} w_{j}=e_{\gamma}\left(\sum_{k=1}^{p} \frac{a_{k}}{\lambda_{j}\left(h_{\beta_{k}}\right)} e_{-\beta_{k}}\right) v_{\lambda_{j}}=\sum_{k=1}^{p}\left(\frac{a_{k}}{\lambda_{j}\left(h_{\beta_{k}}\right)}\left[e_{\gamma}, e_{-\beta_{k}}\right] v_{\lambda_{j}}\right) .
$$

Let us show that each summand of the latter sum equals zero. Assume the converse: $\left[e_{\gamma}, e_{-\beta_{k}}\right] v_{\lambda_{j}} \neq 0$ for some $k \in\{1, \ldots, p\}$. Then by Lemma 1(b) we obtain $\delta=\beta_{k}-\gamma \in \Delta_{+}$. Hence $e_{-\delta} v_{\lambda_{j}} \neq 0$ and $\left\langle\lambda_{j} \mid \delta\right\rangle>0$. On the other hand, from the conditions $\beta_{k}=\gamma+\delta$ and $\gamma \notin \Psi$ it follows that $\delta$ is an active root subordinate to the active root $\beta_{k}$. This means that $\pi\left(\beta_{k}\right) \notin \operatorname{Supp} \delta$. Then by Proposition 5 one has 
$\pi\left(\Psi_{i}\right) \cap \operatorname{Supp} \delta=\varnothing$, whence $\left\langle\lambda_{j} \mid \delta\right\rangle=0$. This contradiction completes Step 3 and the proof of the proposition.

Remark 3. If $p=1$ (that is, $\left|\Psi_{j}\right|=1$ ), then the proof of Proposition 7 considerably simplifies. Namely, in this case there is nothing to prove at Step 1 (since $\mathfrak{n} \cap \mathfrak{u}_{j}=0$ ) whereas Step 2 looses its substantial part: for $i \neq j$ and $\left|\Psi_{i}\right| \geqslant 2$ the condition $\Psi_{i}+\gamma \subset \Psi_{j}$ can hold for no root $\gamma \in \Delta_{+}$. We also note that for $p=1$ the vector $w_{j}$ is proportional to $e_{-\beta_{1}} v_{\lambda_{j}}$.

4.3. In this subsection we prove that the elements of the semigroup $\widehat{\Lambda}_{+}(G / H)$ listed in the statement of Theorem 4 are indecomposable in this semigroup. Since $H$ is connected, in view of Remark 2 it suffices to prove that the non-zero $(B \times H)$-semi-invariant regular functions on $G$ corresponding to the above-mentioned elements are irreducible in $\mathbb{C}[G]$.

For $i=1, \ldots, n$ we denote by $P_{i}$ the function in $\mathbb{C}[G]$ corresponding to the element $v_{\omega_{i}^{*}} \otimes v_{\omega_{i}} \in V\left(\omega_{i}^{*}\right) \otimes V\left(\omega_{i}\right)$ under isomorphism (11). One has $P_{i}(g)=\left\langle v_{\omega_{i}^{*}}, g v_{\omega_{i}}\right\rangle$ for all $g \in G$. The function $P_{i}$ is $(B \times H)$-semi-invariant of weight $\left(\omega_{i}^{*}, \tau\left(\omega_{i}\right)\right)$. Similarly, for $j=1, \ldots, m$ we denote by $Q_{j}$ the function in $\mathbb{C}[G]$ corresponding to the element $v_{\lambda_{j}^{*}} \otimes w_{j} \in V\left(\lambda_{j}^{*}\right) \otimes V\left(\lambda_{j}\right)$ under isomorphism (11). We have $Q_{j}(g)=\left\langle v_{\lambda_{j}^{*}}, g w_{j}\right\rangle$ for all $g \in G$. The function $Q_{i}$ is $(B \times H)$-semi-invariant of weight $\left(\lambda_{j}^{*}, \tau\left(\lambda_{j}\right)-\varphi_{j}\right)$.

Lemma 2. Suppose that $i \in\{1, \ldots, n\}$ and $v \in V\left(\omega_{i}\right) \backslash\{0\}$. Then the function $f \in \mathbb{C}[G]$ corresponding to the element $v_{\omega_{i}^{*}} \otimes v \in V\left(\omega_{i}^{*}\right) \otimes V\left(\omega_{i}\right)$ under isomorphism (11) is irreducible in $\mathbb{C}[G]$.

Proof. Regard the algebra ${ }^{U} \mathbb{C}[G]$. In view of isomorphism (1) we have

$$
U \mathbb{C}[G] \simeq \bigoplus_{\lambda \in \Lambda_{+}(G)} v_{\lambda^{*}} \otimes V(\lambda),
$$

where the component $v_{\lambda^{*}} \otimes V(\lambda)$ is regarded as a subspace in $V\left(\lambda^{*}\right) \otimes V(\lambda)$. The action on the left of the torus $T$ determines a grading on ${ }^{U} \mathbb{C}[G]$ by elements of $\Lambda_{+}(G)$ in such a way that the component of weight $\lambda^{*}$ corresponds to the subspace $v_{\lambda^{*}} \otimes V(\lambda)$. The hypothesis of the lemma implies that $f$ is contained in the component of weight $\omega_{i}^{*}$ of $U \mathbb{C}[G]$. Since the component of weight 0 contains only the constants and the weight $\omega_{i}^{*}$ admits no nontrivial expression as a sum of two elements in $\Lambda_{+}(G)$, it follows that the function $f$ is irreducible in ${ }^{U} \mathbb{C}[G]$. It remains to notice that, for a function in ${ }^{U} \mathbb{C}[G]$, irreducibility in ${ }^{U} \mathbb{C}[G]$ is equivalent to irreducibility in $\mathbb{C}[G]$, since the group $U$ is connected and has no non-trivial characters (see [PV, Theorem 3.17]).

Corollary 2. For all $i=1, \ldots, n$ the function $P_{i}$ is irreducible in $\mathbb{C}[G]$.

We note that for every $i=1, \ldots, n$ the function $P_{i}$ is not only $(B \times H)$-semi-invariant, but also $(B \times B)$-semi-invariant. Therefore the corresponding $(B \times H)$-stable prime divisor $D_{i} \subset G$ is also $(B \times B)$-stable. As was already mentioned in $\S 4.1$ (see Proof 2 of Proposition (6), the complement in $G$ of the open Bruhat cell $G_{0}=B \sigma_{0} B$ is the union of exactly $n$ prime divisors, which are $(B \times B)$-stable. It is clear that these divisors are nothing else but $D_{1}, \ldots, D_{n}$.

To complete the proof of Theorem 4 it remains to prove that the function $Q_{j}$ is irreducible in $\mathbb{C}[G]$ for $j=1, \ldots, m$. 
Proposition 8. For all $i=1, \ldots, n$ and $j=1, \ldots, m$ the function $Q_{j}$ is not divisible by $P_{i}$.

Proof. We fix $i \in\{1, \ldots, n\}, j \in\{1, \ldots, m\}$ and show that $Q_{j}$ is not divisible by $P_{i}$. The function $Q_{j} / P_{i}$ is a $(B \times H)$-semi-invariant rational function on $G$ of weight $\left(\lambda_{j}^{*}-\omega_{i}^{*}, \tau\left(\lambda_{j}-\omega_{i}\right)-\varphi_{j}\right)$. If $i \notin\left\{j_{1}, \ldots, j_{r}\right\}$, then the weight $\lambda_{j}^{*}-\omega_{i}^{*}$ is not dominant, therefore the function $Q_{j} / P_{i}$ is not regular. Taking this into account, further we suppose that $i \in\left\{j_{1}, \ldots, j_{r}\right\}$. Moreover, without loss of generality we may assume $i=j_{1}$. We recall that $w_{j}=f_{j} v_{\lambda_{j}}$, where the element $f_{j}$ is defined in $\S 4.2$. The function corresponding to the element $v_{\lambda_{j}^{*}} \otimes v_{\lambda_{j}}$ under isomorphism (11) is $(B \times H)$-semi-invariant of weight $\left(\lambda_{j}^{*}, \lambda_{j}\right)$. In view of the condition $\operatorname{dim} A\left(\lambda_{j}^{*}, \lambda_{j}\right) \leqslant 1$ we obtain that this function coincides with the function $c P_{i} P_{j_{2}} \ldots P_{j_{r}}$ for some $c \neq 0$. Without loss of generality we assume $c=1$. Then one has $Q_{j}=f_{j}\left(P_{i} P_{j_{2}} \ldots P_{j_{r}}\right)$, where the action on $\mathbb{C}[G]$ of the Lie algebra $\mathfrak{g}$ is induced by the action of $G$ on the right. The element $f_{j}$ acts on the product $P_{i} P_{j_{2}} \ldots P_{j_{r}}$ as a derivation, therefore $Q_{j}=\left(f_{j} P_{i}\right) P_{j_{2}} \ldots P_{j_{r}}+P_{i} \cdot f_{j}\left(P_{j_{2}} \ldots P_{j_{r}}\right)$. Hence the divisibility of $Q_{j}$ by $P_{i}$ is equivalent to the divisibility of $f_{j} P_{i}$ by $P_{i}$. Under isomorphism (1), the function $f_{j} P_{i}$ corresponds to the vector $v_{\omega_{i}^{*}} \otimes\left(f_{j} v_{\omega_{i}}\right) \in V\left(\omega_{i}^{*}\right) \otimes V\left(\omega_{i}\right)$. Let us show that this vector is non-zero, that is, $f_{j} v_{\omega_{i}} \neq 0$. For that, it suffices to find a root $\beta \in \Psi_{j}$ such that $e_{-\beta} v_{\omega_{i}} \neq 0$. The last condition is equivalent to the inequality $\left\langle\omega_{i} \mid \beta\right\rangle>0$, which surely holds for any root $\beta \in \Psi_{j}$ with $\pi(\beta)=\alpha_{i}$. Thus, the vector $f_{j} v_{\omega_{i}} \in V\left(\omega_{i}\right)$ is non-zero. It is easy to see that this vector is not proportional to $v_{\omega_{i}}$. In view of Lemma 2 this implies that $f_{j} P_{i}$ is irreducible in $\mathbb{C}[G]$ and is not proportional to $P_{i}$. Hence $f_{j} P_{i}$ is not divisible by $P_{i}$, and so is $Q_{j}$.

In view of the condition $G \backslash G_{0}=D_{1} \cup \ldots \cup D_{n}$ and Proposition 8 the proof of the irreducibility in $\mathbb{C}[G]$ of each function $Q_{j}$ reduces to the following proposition.

Proposition 9. For every $j=1, \ldots, m$ the restriction of $Q_{j}$ to the open cell $G_{0}$ is an irreducible function in $\mathbb{C}\left[G_{0}\right]$.

Proof. Let $j \in\{1, \ldots, m\}$. Then one has $\lambda_{j}=\omega_{j_{1}}+\ldots+\omega_{j_{r}}$. The restrictions to $G_{0}$ of the functions $P_{1}, \ldots, P_{n}$ are invertible, therefore it suffices to prove that the function $\widehat{Q}_{j}=Q_{j} /\left(P_{j_{1}} \ldots P_{j_{r}}\right)$ is irreducible in $\mathbb{C}\left[G_{0}\right]$. We note that this function is $(B \times H)$ semi-invariant of weight $\left(0,-\varphi_{j}\right)$. Since the group $B \times H$ has an open orbit in $G_{0}$, the subspace in $\mathbb{C}\left[G_{0}\right]$ consisting of all $(B \times H)$-semi-invariant functions of weight $\left(0,-\varphi_{j}\right)$ is one-dimensional. The proof will be completed if we present an irreducible $(B \times H)$ semi-invariant function of weight $\left(0,-\varphi_{j}\right)$ in $\mathbb{C}\left[G_{0}\right]$. The group $U \times N$ is connected and has no non-trivial characters, hence in view of [PV, Theorem 3.17] it suffices to present an irreducible $(T \times S)$-semi-invariant function of weight $\left(0,-\varphi_{j}\right)$ in ${ }^{U} \mathbb{C}\left[G_{0}\right]^{N}$. One has ${ }^{U} \mathbb{C}\left[G_{0}\right]^{N} \simeq{ }^{U} \mathbb{C}\left[U \times T \sigma_{0} \times U\right]^{N} \simeq \mathbb{C}[T \times U / N] \simeq \mathbb{C}[T \times \mathfrak{u} / \mathfrak{n}]$, where the last isomorphism is due to an $S$-equivariant isomorphism $U / N \simeq \mathfrak{u} / \mathfrak{n}$ (see [Mon, lemme 1.4]). The group $T \times S$ acts on the variety $T \times \mathfrak{u} / \mathfrak{n}$ as follows: any pair $(t, s) \in T \times S$ takes each pair $\left(t_{0}, x\right) \in T \times \mathfrak{u} / \mathfrak{n}$ to $\left(t t_{0}\left(\sigma_{0} s^{-1} \sigma_{0}^{-1}\right), s x\right)$. We recall that by Theorem 3 the subspace $\mathfrak{u} / \mathfrak{n}$ is isomorphic as an $S$-module to the direct sum $\mathbb{C}_{\varphi_{1}} \oplus \ldots \oplus \mathbb{C}_{\varphi_{m}}$, where $\mathbb{C}_{\varphi_{i}} \simeq \mathbb{C}$ is the subspace of weight $\varphi_{i}$ with respect to $S$. Let $l_{j}$ denote the $j$ th coordinate function on $\mathfrak{u} / \mathfrak{n}$. Being a linear function, $l_{j}$ is an irreducible $S$-semi-invariant function of weight $-\varphi_{j}$. Hence the function $1 \otimes l_{j} \in \mathbb{C}[T] \otimes \mathbb{C}[\mathfrak{u} / \mathfrak{n}] \simeq \mathbb{C}[T \times \mathfrak{u} / \mathfrak{n}]$ is an irreducible $(T \times S)$-semi-invariant function of weight $\left(0,-\varphi_{j}\right)$. 


\section{REFERENCES}

[Av1] R.S. Avdeev, Extended weight semigroups of affine spherical homogeneous spaces of nonsimple semisimple algebraic groups, Izv. math., 74 (2010), no. 6, 1103-1126; Russian original: Р.С. Авдеев, Расширенные полугруппы старших весов аффинных сферических однородных пространств непростых полупростых алгебраических групп, Изв. РАН. Сер. матем., 74:6 (2010), 3-26; see also arXiv:math.RT/1012.0132.

[Av2] R. S. Avdeev, On solvable spherical subgroups of semisimple algebraic groups, Trans. Mosc. math. soc., 2011, 1-44; Russian original: Р. С. Авдеев, О разрешимых сферических подгруппах полупростых алгебраических групn, Труды Моск. матем. общ-ва, 72:1 (2011), 5-62; see also arXiv:math.GR/1102.4595.

[Gor] N.E. Gorfinkel, Harmonic analysis on a class of spherical homogeneous spaces, Math. Notes, 90 (2011), no. 5, 678-685; Russian original: Н.Е. Горфинкель, Гармонический анализ на некотором классе сферических однородных пространств, Матем. заметки, 90:5 (2011), 703711.

[Kn] F. Knop, Weylgruppe und Momentabbildung, Invent. Math., 99 (1990), 1-23.

[Kr] M. Krämer, Sphärische Untergruppen in kompakten zusammenhängenden Liegruppen, Compositio Math., 38 (1979), no. 2, 129-153.

[Mon] P.-L. Montagard, Une nouvelle propriété de stabilité du pléthysme, Comment. Math. Helvetici, 71 (1996), 475-505.

[Pa1] D. I. Panyushev, Complexity and rank of homogeneous spaces, Geometriae Dedicata, 34 (1990), no. 3, 249-269.

[Pa2] D. I. Panyushev, Complexity and nilpotent orbits, Manuscripta math., 83 (1994), 223-237.

[Pop] V.L. Popov, Picard groups of homogeneous spaces of linear algebraic groups and one-dimensional homogeneous vector bundles, Math. USSR-Izv., 8 (1974), no. 2, 301-327; Russian original: В.Л. Попов, Группы Пикара однородных пространств линейных алгебрачческих групп и одномерные однородные векторные расслоения, Изв. АН СССР. Сер. матем., 38:2 (1974), 294-322.

[PV] V.L. Popov, E. B. Vinberg, Invariant theory, Algebraic geometry. IV: Linear algebraic groups, invariant theory, Encyclopaedia of Mathematical Sciences, vol. 55, 1994, 123-278; Russian original: Э. Б. Винберг, В. Л. Попов, Теория инвариантов, в кн.: Алгебраическая геометрия - 4, Итоги науки и техн. Сер. Соврем. пробл. мат. Фундам. направления, 55, ВИНИТИ, М., 1989, 137-309.

[Tim] D. A. Timashev, Homogeneous spaces and equivariant embeddings, Encyclopaedia of Mathematical Sciences, vol. 138, Springer-Verlag, Berlin Heidelberg 2011.

[VK] E. B. Vinberg, B. N. Kimelfeld, Homogeneous domains on flag manifolds and spherical subgroups of semisimple Lie groups, Funct. Anal. Appl., 12 (1978), no. 3, 168-174; Russian original: Э. Б. Винберг, Б. Н. Кимельфельд, Однородные области на флаговых многообразиях и сферические подгруппы полупростых групп Ли, Функц. анализ и его прилож., 12:3 (1978), $12-19$.

Chair of Higher Algebra, Department of Mechanics and Mathematics, Moscow State University, 1, Leninskie Gory, Moscow, 119992, Russia

E-mail address: suselr@yandex.ru, nataly.gorfinkel@gmail.com 